\title{
Towards Identifying Potential Research Collaborations from Scientific Research Networks using Scholarly Data
}

\author{
Yanet Garay \\ Department of Computer Science \\ and Cyber-ShARE \\ University of Texas at El Paso, El \\ Paso, Texas, USA \\ ydgaray@miners.utep.edu
}

\author{
Monika Akbar \\ Department of Computer Science \\ and Cyber-ShARE \\ University of Texas at El Paso, El \\ Paso, Texas, USA \\ makbar@utep.edu
}

\author{
Ann Q. Gates \\ Department of Computer Science and \\ Cyber-ShARE \\ University of Texas at EI Paso, El Paso, \\ Texas, USA \\ agates@utep.edu
}

\begin{abstract}
Identifying research areas of researchers is a difficult task because of the various levels of abstraction in which information may be stored; however, such a task is essential for detecting potential research collaborations within an institution. This work describes an approach to create a scientific research network with topics identified from the researchers' scholarly data and relations between topics by analyzing data harvested from digital libraries and queries to domain ontologies. The relations are used to connect the researchers. Such networks have the potential for revealing the synergy between different topics and researchers within an institution. It will also show less explored research areas that can be targeted for further study. The poster will describe the approach and how it was applied to a biomedical domain at the university.
\end{abstract}

\section{Keywords}

Scholarly data; scientific research network; research collaborations.

\section{INTRODUCTION}

Gaining a better understanding of the current state of research within an institution is critical for its advancement towards its vision. In order to support collaborative and interdisciplinary work within and across academic institutions, it is vital to be able to curate and analyze scholarly data [5]. Institutional repositories have become a reliable medium for sharing scholarly work [2]. Study of scientific collaboration networks has been a field of intense interest in recent years because it not only depicts academic interactions and contributions, but also represents the knowledge structure in scientific communities [1][4].

The poster will describe the approach to construct a scientific research network (SRN) based on data harvested from metadata of scientific publications and academic activities by using domainspecific ontologies to identify and connect the researchers. The SRN can serve as the foundation for identifying possible research collaborations and new research directions. The construction of the SRN has three principal goals: 1) identify and represent individual researchers' research focus; 2) detect connections between researchers, and 3) discover potential research directions.

Permission to make digital or hard copies of part or all of this work for personal or classroom use is granted without fee provided that copies are not made or distributed for profit or commercial advantage and that copies bear this notice and the full citation on the first page. Copyrights for thirdparty components of this work must be honored. For all other uses, contact the Owner/Author.

Copyright is held by the owner/author(s).

JCDL '16, June 19-23, 2016, Newark, NJ, USA

ACM 978-1-4503-4229-2/16/06.

DOI: http://dx.doi.org/10.1145/2910896.2925439
The poster will describe and elucidate the approach by demonstrating how it was applied to a group of researchers at the University of Texas at El Paso (UTEP).

\section{PROBLEM FORMULATION}

Discovering potential research collaborations has traditionally been done by searching publications based on user-defined keywords. These keywords are defined by authors and aim at facilitating discovery by condensing research work into a few words. However, one of the challenges in this approach is that keywords are defined at different levels of granularity i.e., some keywords may be too abstract or too specific, making it difficult to 1) match keywords to identify related and potential research collaborations, 2) discover the main research area of a researcher and 3) trace how researcher's interests have changed over time, and 4) determine what the main areas of research of an organization are based on the publications of its researchers.

\section{APPROACH}

The approach constructs SRNs by analyzing data harvested from metadata of academic and research activities in a biomedical domain and by querying domain-specific ontologies to discover potential research collaborations.

The first step in the approach is to extract research publication and academic activity data. At UTEP, this was accomplished using Digital Measures (DM), a web-based data repository of faculty accomplishments. The effort targeted 92 faculty members from the biomedical research areas. For each of these faculties, a keyword list was constructed containing single-word keywords and two-word keywords. The next step is to calculate the keyword frequency by counting the number of times the keyword appears in the researcher's data from DM. To identify paths that link two or more keywords together, Ontobee [3] was used. Ontobee is a linked data server designed for ontologies that combines two basic features in the ontology-term search: 1) a web interface for displaying the search result details including hierarchy for a specific ontology term; and 2) a RDF/XML representation for ontology terms corresponding to the HTML web page results that can be accessed by Semantic Web applications. Ontobee provides access to ontologies covering domains such as: anatomy, health and experiments [3]. For this work, Ontobee is used to determine if the keywords included in the keyword list belong to a specific domain. This search allows us to discard words that were included in the keyword list, but should not be considered as keywords. On the other hand, there is a small possibility that a keyword would not be found in Ontobee, but should still be considered as a keyword. Once the keyword is determined to belong to the specific domain, the following is retrieved from Ontobee 1) the parent concept of the keyword, i.e., the immediate top level concept on the ontology hierarchy; 2) the asserted axioms of the keyword; and 3) the asserted axioms of the parent concept. 
The semantic of the connection between a keyword and the parent concept can be read as an is a relationship. The asserted axioms are utilized to determine if the keyword is part of a more general concept. The concepts extracted from Ontobee will be included in the SRN of each faculty.

Using the approach described above, a method was created to generate the SRN representing the researcher's interests as a directed weighted graph. The directed edges in the graph are used to identify the source and target nodes. Similarly the weighted edges are used to classify the relationships between the nodes: is a $=1$ (strong), is part of $=5$ (less strong). The relation is $a$ is considered to be more significant in the semantic of the SRN than relation part of. A keyword that is a concept $\mathrm{X}$ has a stronger relation with $\mathrm{X}$ than a keyword that is part of $\mathrm{X}$. To determine how strong a relationship between two nodes is, the total weight of the path connecting the nodes is calculated. A smaller weight path signifies that the relationship is stronger. The method steps are described below:

1) For each faculty, compute their own keyword list consisting of nested pairs (keyword, frequency).

2) For each keyword $k_{i}$ in the keyword list that is found in Ontobee, a node is created. The asserted axioms, the parent concept, and the asserted axioms of the parent concept are retrieved.

2.1 The relationship $k_{i}$ is a Concept $A$ (parent concept) is obtained using the hierarchy of the ontology. Concept $A$ is included as a node and an edge with the weight of 1 is created pointing to Concept $A$.

2.2 The asserted axioms are used to look for the relationships a) Concept $A$ is part of Concept B; and b) $k_{i}$ is part of Concept $C$. Concept $B$ and $C$ are included as nodes and the respectively relationships with Concept $A$ and $k_{i}$ are created with a weight of 5 .

3) If two nodes are connected by an edge with a weight of 1(A is $a \mathrm{~B}$ ), the direction of the edge (extracted from the concept hierarchy in Ontobee) will indicate which general concept will have its frequency incremented. The reasoning behind this is that the relationship A is a B implies that where $\mathrm{A}$ has been mentioned, $\mathrm{B}$ is also mentioned.

To illustrate, we apply the algorithm to the biomedical domain: Consider Faculty X whose keyword list is composed of nested pairs (keyword, weight) as follows: $\mathrm{K}=\{(\mathrm{LH}, 51)$, (hypothalamus, 42), (PVH, 69) $\}$. After applying Step 2 of the algorithm the parent concept Regional part of the brain was obtained and the following relationships were extracted: 1) LH is a Regional part of the brain; 2) hypothalamus is a Regional part of the brain; and 3) PVH is a Regional part of the brain. After applying Step 3, the following is the updated keyword list: $\mathrm{K}=\{(\mathrm{LH}, 51)$, (hypothalamus, 110), (PVH, 69), (Regional part of the brain, 230)\} Fig. 1 shows eight keywords (in color green) and six ontological concepts (in color gray) that were found by querying Ontobee. In addition, the semantics of the connections and paths between keywords and concepts is depicted. After applying the proposed algorithm, the keywords Hypothalamic and Hindbrain were extracted in the keyword list for Faculty X Ontobee was queried and a partial graph was built showing that the two keywords are related at a higher level by the concepts Brain and Central Nervous System if the next paths are followed:

Hypothalamic is part of Hypothalamus is a Regional part of the brain is part of Brain is part of Central Nervous System; Hindbrain is part of Brain is part of Central Nervous System.

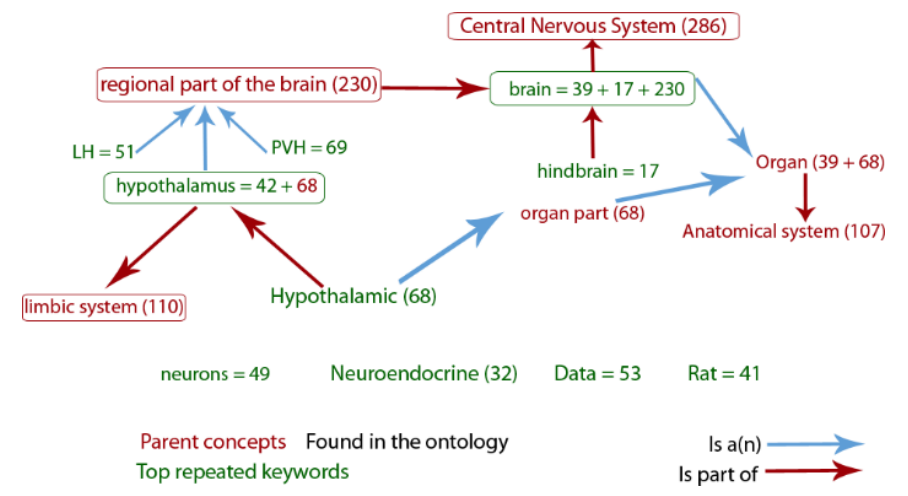

Figure 1. Partial graph for faculty $X$

The last step is to combine all the individual networks into one SRN. The overlapped nodes indicate a connection between scholars and nodes where the highest frequencies indicate the main research focus for the group of faculty.

\section{CONCLUSION}

This work proposes the preliminary work in defining an approach to construct SRNs. The approach is based on analyzing data harvested from metadata of scientific publications and querying ontologies to identify and connect the researchers. The work investigates detecting connections between researchers beyond the limitations depending on keywords selected by the research authors. Mapping researchers' scholarship using more general topics and more specifics allows detecting a wider range of connections. Future work includes completing the development of a tool that implements the described approach. Also the creation of a new method to detect the topics that are less explored in the SRN that could become new potential research directions is planned. This method will help in identify the expertise that could potentially be interested in focusing on these less explored topics. In addition, validation of the results with domain experts needs to be conducted to show the effectiveness of the approach.

\section{ACKNOWLEDGMENTS}

This work is supported in part by the National Science Foundation (NSF) grants HRD-1242122 and DUE-0963648. Any opinions, findings, and conclusions or recommendations expressed in this paper are those of the author(s) and do not necessarily reflect the views of the NSF.

\section{REFERENCES}

[1] Newman, M. E. 2004. Coauthorship networks and patterns of scientific collaboration. Proceedings of the national academy of sciences, 101(suppl 1), 5200-5205.

[2] Schlangen, M. 2015. Content, Credibility, and Readership: Putting Your Institutional Repository on the Map. Public Services Quarterly, 11(3), 217-224.

[3] Xiang, Z., Mungall, C., Ruttenberg, A., \& He, Y. 2011. Ontobee: A Linked Data Server and Browser for Ontology Terms. In ICBO.

[4] Yang, Y., Man Au Yeung, C., Weal, M., \& Davis, H. 2009. The researcher social network: A social network based on metadata of scientific publications. In: Proceedings of the WebSci'09: Society On-Line, (18-20 March 2009, Greece).

[5] Young, L., Denize, S., Simoff, S., Nankani, E., \& Wilkinson, I. 2015. Researching The Structures and Processes of Collaborative Academic Networks. In Proceedings of the 2010 Academy of Marketing Science (AMS) Annual Conference (pp. 112-116). Springer International Publishing. 